\title{
SMALE'S PROBLEM FOR CRITICAL POINTS ON CERTAIN TWO RAYS
}

\author{
AIMO HINKKANEN ${ }^{\bowtie}$ and ILGIZ KAYUMOV
}

\author{
(Received 27 October 2009; accepted 4 January 2010)
}

Communicated by P. C. Fenton

\begin{abstract}
Let $f$ be a polynomial of degree $n \geq 2$ with $f(0)=0$ and $f^{\prime}(0)=1$. We prove that there is a critical point $\zeta$ of $f$ with $|f(\zeta) / \zeta| \leq 1 / 2$ provided that the critical points of $f$ lie in the sector $\left\{r e^{i \theta}: r>0,|\theta| \leq \pi / 6\right\}$, and $|f(\zeta) / \zeta|<2 / 3$ if they lie in the union of the two rays $\left\{1+r e^{ \pm i \theta}: r \geq 0\right\}$, where $0<\theta \leq \pi / 2$.
\end{abstract}

2000 Mathematics subject classification: primary 30C10; secondary 30C15.

Keywords and phrases: polynomials, critical points, Smale's problem.

\section{Introduction and results}

In 1981, Smale [13] made the following well-known conjecture. Let $f$ be a polynomial of degree $n \geq 2$ normalized by $f(0)=0$ and $f^{\prime}(0)=1$. Unless otherwise mentioned, we assume that our polynomials $f$ satisfy these normalizations. Let us set

$$
S(f)=\min \left\{\left|\frac{f(\zeta)}{\zeta}\right|: f^{\prime}(\zeta)=0\right\}
$$

and

$$
K_{n}=\sup \left\{S(f): \operatorname{deg} f=n, f(0)=0, f^{\prime}(0)=1\right\} .
$$

The conjecture is that then $K_{n}=1-1 / n$. Smale proved that $S(f)<4$ for all such $f$. If $f(z)=z+c z^{n}$ for some nonzero $c$, then $S(f)=1-1 / n$. Thus

$$
1-\frac{1}{n} \leq K_{n} \leq 4
$$

This material is based upon work supported by the National Science Foundation under grant no. 0758226. The second author was supported by RFBR (grant nos 08-01-00381, 09-01-12188 ofi-m) and by the Russian Federal Agency of Education, grant no. P 944.

(C) 2010 Australian Mathematical Publishing Association Inc. 1446-7887/2010 \$16.00 
Many improvements have been obtained since, either for all $f$ or for $f$ in special classes of functions. Nonetheless, the number 4 remains the best known upper bound that is an absolute constant and is applicable to all $f$.

It was proved by Sikorav (see [14]) that if $n \leq 4$, then $K_{n}=1-1 / n$, and furthermore that

$$
\left|\frac{f(\zeta)}{\zeta}\right|<1-\frac{1}{n}
$$

for some critical point $\zeta$ of $f$, that is, a zero of $f^{\prime}$, unless $f(z)=z+c z^{n}$. Numerical experiments by Marinov and Sendov [9] suggest that the same conclusion holds when $n \leq 10$.

Beardon et al. [1] obtained $K_{n} \leq 4^{1-1 / n}$, which was slightly improved in [2, 7]. The best known upper bound is due to Crane (see $[4,5]$ ). If the critical points of $f$ all have the same modulus, or if the values of $f$ at the critical points have equal modulus, then a theorem of Sheil-Small [11, pp. 361-362], whose proof uses the method of Córdova and Rusheweyh [3], shows that $S(f)<1$. Dubinin [6] improved this to $S(f) \leq 1-1 / n$ when the critical points have equal modulus. Tischler [14] proved $S(f)<1$ when the nonzero zeros of $f$ have equal modulus.

In the case where $f$ has only real zeros, Palais (see [12, p. 159]) proved that $S(f)<1$ while Tischler [14] obtained $S(f) \leq 1-1 / n$. If $f$ has only real zeros, it follows from Rolle's theorem that $f^{\prime}$ has only real zeros. Since the converse does not hold, the case where $f$ has only real critical points is more general than that where $f$ has only real zeros.

Under the assumption that $f$ has only real critical points, Sheil-Small [11, p. 368] obtained $S(f)<e-2$ while Rahman and Schmeisser [10, p. 217] proved the slightly better result

$$
S(f) \leq \frac{n-2}{n}\left(\left(\frac{n-1}{n-2}\right)^{n-1}-2\right)<e-2,
$$

where $n \geq 3$.

Suppose that the critical points of $f$ are contained in the union of $k$ rays from the origin to infinity. In [8] we conjectured that for such a function $f$, we have $S(f) \leq 1-1 /(k+1)$, which would imply that $K_{n}=1-1 / n$, and proved that this is true for $k=1$ and $k=2$.

The original question of Smale did not initially have the above normalization. So let us now assume merely that $f$ is a polynomial of degree $n \geq 2$. Pick any $t$ in the complex plane $\mathbb{C}$ such that $f^{\prime}(t) \neq 0$. Then we consider

$$
S(f, t)=\min \left\{\left|\frac{f(\zeta)-f(t)}{(\zeta-t) f^{\prime}(t)}\right|: f^{\prime}(\zeta)=0\right\} .
$$

Since we may replace $f$ by $a f+b$ and the variable $z$ by $c z+d$ for any complex numbers $a, b, c, d$ with $a c \neq 0$, it is easily seen that $S(f, t)=S(g, 0)$ for a polynomial $g$ of degree $n$ with $g(0)=0$ and $g^{\prime}(0)=1$. Namely, we may take

$$
g(z)=(f(\beta z+t)-f(t)) /\left(\beta f^{\prime}(t)\right) \quad \forall \beta \in \mathbb{C} \backslash\{0\} .
$$


However, the critical points of $g$ are not at the same locations as those of $f$, so if those of $f$ are taken to lie in the union of $k$ rays from the particular point 0 to infinity, then those of $g$ will lie in the union of $k$ rays merely from some point $\alpha$ (usually not 0 ) to infinity. Note that if $\alpha \neq 0$, by an appropriate choice of $\beta$, we may assume that $\alpha=1$.

In this paper we only consider the case where $k=1$ or $k=2$. If $\alpha=0$ after passing to $g$, our problem has already been solved in [8]. Therefore we limit ourselves to the case where $\alpha \neq 0$.

Suppose that $k=1$, so that there is only one ray which is part of a certain straight line, or that $k=2$ and the two rays form a straight line. If this line passes through the origin, then again we are reduced to the case covered in [8]. Therefore we will assume that under these circumstances this line does not go through the origin. Then we may replace $\alpha$ by that point on the line that is closest to the origin. By a further rotation and dilation, we may then assume that $\alpha=1$ and that the line is vertical. We will henceforth take these comments as understood.

In this paper we study the problem of estimating $S(g, 0)$ when the critical points of $g$ lie in the union of one or two rays from a finite point to infinity, and in one case the same problem when the critical points lie in a certain sector. Now, after explaining the motivation for our study, we revert back to writing $f$ instead of $g$ and assume again that $f$ is normalized by $f(0)=0$ and $f^{\prime}(0)=1$.

If $n=5$ and the critical points of $f$ are at \pm 1 and $\pm i$, then $S(f)=4 / 5$. By moving the critical point at $z=1$ slightly to obtain a new normalized polynomial $f_{1}$, we can still arrange to have $S(f)$ arbitrarily close to $4 / 5$. Then the line $L_{1}$ containing the points -1 and $i$ and the line $L_{2}$ containing $-i$ and the new critical point close to 1 but not on the line from $-i$ to 1 will intersect at a point $\alpha$. Thus the critical points of $f_{1}$ lie in the union of two rays from $\alpha$ to infinity. This shows that the best constant when $k=2$ is at least $4 / 5$. In this paper, when we take $k=2$, we impose further restrictions on the configuration of the two rays and obtain a better estimate in those cases.

We prove the following results.

THEOREM 1.1. Let $f$ be a polynomial of degree $n \geq 2$ with $f(0)=0$ and $f^{\prime}(0)=1$. Suppose that the critical points of $f$ lie in the sector $\left\{r e^{i \theta}: r>0,|\theta| \leq \pi / 6\right\}$. Then $S(f) \leq 1 / 2$, and equality holds if, and only if, $n=2$.

THEOREM 1.2. Let $f$ be a polynomial of degree $n \geq 2$ with $f(0)=0$ and $f^{\prime}(0)=1$. Suppose that the critical points of $f$ lie on the ray $\left\{1+r e^{i \theta}: r \geq 0\right\}$, where $0 \leq \theta \leq$ $\pi / 2$. Then $S(f) \leq 1 / 2$, and equality holds if, and only if, $n=2$.

THEOREM 1.3. Let $f$ be a polynomial of degree $n \geq 2$ with $f(0)=0$ and $f^{\prime}(0)=1$. Suppose that the critical points of $f$ lie in the union of the rays $\left\{1+r e^{ \pm i \theta}: r \geq 0\right\}$, where $0<\theta \leq \pi / 2$. Then $S(f)<2 / 3$.

Of course, if $0 \leq \theta \leq \pi / 6$ in Theorem 1.3, then we are in a situation already covered by Theorem 1.1 or 1.2 , in which case one of those theorems yields the upper bound $1 / 2$ instead of $2 / 3$.

The proofs use methods similar to those introduced by us in [8]. 


\section{Proof of Theorem 1.1}

Let the assumptions of Theorem 1.1 be satisfied. Let the critical points of $f$ be denoted by $z_{j}$ where $1 \leq j \leq n-1$, listing each critical point according to its multiplicity. Let $z_{1}$ be a critical point of $f$ with minimal modulus. As in [8], we may write

$$
\frac{f(z)}{z}=\int_{0}^{1} \prod_{j=1}^{n-1}\left(1-\frac{t z}{z_{j}}\right) d t
$$

Hence

$$
\left|\frac{f\left(z_{1}\right)}{z_{1}}\right| \leq \int_{0}^{1}(1-t) \prod_{j=2}^{n-1}\left|1-\frac{t z_{1}}{z_{j}}\right| d t .
$$

When $2 \leq j \leq n-1$,

$$
\left|1-\frac{t z_{1}}{z_{j}}\right| \leq 1
$$

To see this, it suffices to prove that $\left|1-t z_{1} / z_{j}\right|^{2} \leq 1$, which is equivalent to

$$
t^{2}\left|\frac{z_{1}}{z_{j}}\right|^{2} \leq 2 t \operatorname{Re} \frac{z_{1}}{z_{j}}=2 t\left|\frac{z_{1}}{z_{j}}\right| \cos (\theta-\psi),
$$

where we have written $z_{1}=\left|z_{1}\right| e^{i \theta}$ and $z_{j}=\left|z_{j}\right| e^{i \psi}$. Here $|\theta| \leq \pi / 6$ and $|\psi| \leq \pi / 6$. Now $0<\left|z_{1}\right| \leq\left|z_{j}\right|$ and $|\theta-\psi| \leq \pi / 3$ so that $\cos (\theta-\psi) \geq 1 / 2$. This implies (2.2).

It follows that

$$
\left|\frac{f\left(z_{1}\right)}{z_{1}}\right| \leq \int_{0}^{1}(1-t) d t=\frac{1}{2} .
$$

The equality $\left|f\left(z_{1}\right) / z_{1}\right|=1 / 2$ can hold only if equality holds in (2.2) for all $t \in(0,1)$, which is never the case, or if $n-1=1$. Thus equality holds at most when $n=2$. On the other hand, if $n=2$, it is easily seen that $\left|f\left(z_{1}\right) / z_{1}\right|=1 / 2$. This completes the proof of Theorem 1.1.

\section{Proof of Theorem 1.2}

Let the assumptions of Theorem 1.2 be satisfied. Let the critical points of $f$ be denoted by $z_{j}$ where $1 \leq j \leq n-1$, listing each critical point according to its multiplicity. Let $z_{1}$ be a critical point of $f$ with minimal modulus. Then, if we write $z_{j}=1+r_{j} e^{i \theta}$, we have $0 \leq r_{1} \leq r_{j}$ if $2 \leq j \leq n-1$. We again apply (2.1). Now we need to estimate

$$
\left|1-\frac{t z_{1}}{z_{j}}\right|=\left|1-t \frac{1+r_{1} e^{i \theta}}{1+r_{j} e^{i \theta}}\right| .
$$


We have, when $0<t \leq 1$,

$$
\begin{aligned}
t^{-1}\left(\left|1-t \frac{1+r_{1} e^{i \theta}}{1+r_{j} e^{i \theta}}\right|^{2}-1\right) & =t\left|\frac{1+r_{1} e^{i \theta}}{1+r_{j} e^{i \theta}}\right|^{2}-2 \operatorname{Re} \frac{1+r_{1} e^{i \theta}}{1+r_{j} e^{i \theta}} \\
& \leq\left|\frac{1+r_{1} e^{i \theta}}{1+r_{j} e^{i \theta}}\right|^{2}-2 \operatorname{Re} \frac{1+r_{1} e^{i \theta}}{1+r_{j} e^{i \theta}}
\end{aligned}
$$

The last quantity multiplied by $\left|1+r_{j} e^{i \theta}\right|^{2}$ is equal to

$$
\begin{aligned}
\mid 1+ & \left.r_{1} e^{i \theta}\right|^{2}-2 \operatorname{Re}\left(1+r_{1} e^{i \theta}\right)\left(1+r_{j} e^{-i \theta}\right) \\
& =1+r_{1}^{2}+2 r_{1} \cos \theta-2\left(1+r_{1} r_{j}+\left(r_{1}+r_{j}\right) \cos \theta\right) \\
& =-1+r_{1}\left(r_{1}-2 r_{j}\right)-2 r_{j} \cos \theta<0
\end{aligned}
$$

since $0<r_{1} \leq r_{j}$ and $\cos \theta \geq 0$. This proves that

$$
\left|1-\frac{t z_{1}}{z_{j}}\right| \leq 1
$$

when $0 \leq t \leq 1$. In the same way as in the proof of Theorem 1.1, it now follows that $\left|f\left(z_{1}\right) / z_{1}\right| \leq 1 / 2$, with equality if, and only if, $n=2$. This completes the proof of Theorem 1.2.

\section{Proof of Theorem 1.3}

The idea of the proof of Theorem 1.3 is to reduce the situation to that considered in [8]. There we obtained the following result (see [8, (4)]).

Lemma 4.1. Suppose that $A$ and $u$ are real numbers with $u \geq 1$. Define

$$
h_{1}(u, A)=\int_{0}^{1}(1-t)\left(1+\frac{t}{u}\right) e^{A t / u} d t
$$

and

$$
h_{2}(u, A)=\int_{0}^{1}(1-t)(1+t u) e^{-A t} d t .
$$

Then

$$
\min \left\{h_{1}(u, A), h_{2}(u, A)\right\} \leq 2 / 3 .
$$

Equality holds if, and only if, $A=0$ and $u=1$.

Let the assumptions of Theorem 1.3 be satisfied. Let the critical points of $f$ be denoted by $z_{j}=1+r_{j} e^{ \pm i \theta}$, where $1 \leq j \leq n-1$ and $r_{j} \geq 0$, listing each critical point according to its multiplicity. If all critical points lie on one of the two rays, a better conclusion with less than $2 / 3$ replaced by at most $1 / 2$ follows from Theorem 1.2. Therefore we may assume that each of the two rays contains a critical point of $f$. Hence $n \geq 3$. 
If $z_{1}=1$ is a critical point of $f$, the proof is easier, for now

$$
|f(1)| \leq \int_{0}^{1}(1-t) \prod_{j=2}^{n-1}\left|1-\frac{t}{z_{j}}\right| d t
$$

and when $2 \leq j \leq n-1$ and $0<t \leq 1$,

$$
\begin{aligned}
\frac{\left|z_{j}\right|^{2}}{t}\left(\left|1-\frac{t}{z_{j}}\right|^{2}-1\right) & =t-2 \operatorname{Re}\left(z_{j}\right) \\
& \leq 1-2\left(1+r_{j} \cos \theta\right)=-1-2 r_{j} \cos \theta<0
\end{aligned}
$$

since $\cos \theta>0$. Hence $\left|1-t / z_{j}\right|<1$, so that we obtain $|f(1)| \leq 1 / 2$ in the same way as in the proof of Theorem 1.1. Thus we assume from now on that $f^{\prime}(1) \neq 0$.

We label the critical points of $f$ so that $z_{j}=1+r_{j} e^{i \theta}$ when $1 \leq j \leq k-1$ and $z_{j}=1+r_{j} e^{-i \theta}$ when $k \leq j \leq n-1$, where $2 \leq k \leq n-1$, and in addition so that $0<r_{1} \leq r_{2} \leq \cdots \leq r_{k-1}$ and $0<r_{k} \leq r_{k+1} \leq \cdots \leq r_{n-1}$.

We seek to prove that

$$
\min \left\{\left|\frac{f\left(z_{1}\right)}{z_{1}}\right|,\left|\frac{f\left(z_{k}\right)}{z_{k}}\right|\right\} \leq \frac{2}{3} .
$$

After proving (4.3), we will discuss the possible cases of equality.

To complete the proof of Theorem 1.3, we need the following two lemmas.

LEMma 4.2. Suppose that $0<r \leq \rho,|\theta| \leq \pi / 2$, and $0 \leq t \leq 1$. Then

$$
\left|1-t \frac{1+r e^{i \theta}}{1+\rho e^{i \theta}}\right| \leq 1-t \frac{r}{\rho} .
$$

LEMMA 4.3. Suppose that $r>0, \rho>0,|\theta| \leq \pi / 2$, and $0 \leq t \leq 1$. Then

$$
\left|1-t \frac{1+r e^{i \theta}}{1+\rho e^{-i \theta}}\right| \leq 1+t \frac{r}{\rho} .
$$

Proof OF Lemma 4.2. If $0<t \leq 1$, then $t r / \rho \leq 1$, so that

$$
\begin{aligned}
\frac{\left|1+\rho e^{i \theta}\right|^{2} \rho^{2}}{t} & \left(\left|1-t \frac{1+r e^{i \theta}}{1+\rho e^{i \theta}}\right|^{2}-\left(1-t \frac{r}{\rho}\right)^{2}\right) \\
= & t\left(\left|1+r e^{i \theta}\right|^{2} \rho^{2}-\left|1+\rho e^{i \theta}\right|^{2} r^{2}\right) \\
& \quad-2 \operatorname{Re}\left(\left(1+r e^{i \theta}\right)\left(1+\rho e^{-i \theta}\right) \rho^{2}-r \rho\left|1+\rho e^{i \theta}\right|^{2}\right) \\
= & t(\rho-r)(\rho+r+2 r \rho \cos \theta)-2\left(\rho^{2}+(\rho-r) \rho^{2} \cos \theta\right) \\
\leq & \left(\rho^{2}-r^{2}+2 r \rho(\rho-r) \cos \theta\right)-2 \rho^{2}(1+(\rho-r) \cos \theta) \\
= & -r^{2}-\rho^{2}-2 \rho(\rho-r)^{2} \cos \theta<0 .
\end{aligned}
$$

This proves (4.4) and hence Lemma 4.2. 
Proof OF LEMMA 4.3. If $0<t \leq 1$, then

$$
\begin{aligned}
& \frac{\left|1+\rho e^{-i \theta}\right|^{2} \rho^{2}}{t}\left(\left|1-t \frac{1+r e^{i \theta}}{1+\rho e^{-i \theta}}\right|^{2}-\left(1+t \frac{r}{\rho}\right)^{2}\right) \\
& =t\left(\left|1+r e^{i \theta}\right|^{2} \rho^{2}-\left|1+\rho e^{-i \theta}\right|^{2} r^{2}\right) \\
& \quad-2 \operatorname{Re}\left(\left(1+r e^{i \theta}\right)\left(1+\rho e^{i \theta}\right) \rho^{2}+r \rho\left|1+\rho e^{-i \theta}\right|^{2}\right) \\
& =t(\rho-r)(\rho+r+2 r \rho \cos \theta) \\
& \quad-2\left(\rho^{2}+r \rho+r \rho^{3}(1+\cos 2 \theta)+\left((r+\rho) \rho^{2}+2 r \rho^{2}\right) \cos \theta\right) .
\end{aligned}
$$

If $r \geq \rho$ then the quantity in (4.6) is negative.

If $r<\rho$ then the quantity in (4.6) does not exceed

$$
\begin{aligned}
(\rho-r)(\rho+r+2 r \rho \cos \theta) & \\
& -2 \rho\left(\rho+r+r \rho^{2}(1+\cos 2 \theta)+((r+\rho) \rho+2 r \rho) \cos \theta\right) \\
=- & (\rho+r)^{2}-2 r \rho^{3}(1+\cos 2 \theta)+2 \rho((\rho-r) r-(3 r+\rho) \rho) \cos \theta \\
= & (\rho+r)^{2}-2 r \rho^{3}(1+\cos 2 \theta)+2 \rho\left(-r^{2}-\rho^{2}-2 r \rho\right) \cos \theta<0 .
\end{aligned}
$$

This completes the proof of Lemma 4.3.

We now return to the proof of Theorem 1.3. Since our problem does not change if we replace the polynomial $f$ by $\overline{f(\bar{z})}$, we may assume that $r_{1} \leq r_{k}$. Note that $1+x \leq e^{x}$ for all real $x$. We have

$$
\left|\frac{f\left(z_{1}\right)}{z_{1}}\right| \leq \int_{0}^{1}(1-t) \prod_{j=2}^{n-1}\left|1-\frac{t z_{1}}{z_{j}}\right| d t .
$$

Applying Lemma 4.2 with $r=r_{1}$ and $\rho=r_{j}$ when $2 \leq j \leq k-1$, and applying Lemma 4.3 with $r=r_{1}$ and $\rho=r_{j}$ when $k \leq j \leq n-1$, we obtain

$$
\begin{aligned}
\left|\frac{f\left(z_{1}\right)}{z_{1}}\right| & \leq \int_{0}^{1}(1-t) \prod_{j=2}^{k-1}\left(1-\frac{t r_{1}}{r_{j}}\right) \prod_{j=k}^{n-1}\left(1+\frac{t r_{1}}{r_{j}}\right) d t \\
& \leq \int_{0}^{1}(1-t)\left(1+\frac{t r_{1}}{r_{k}}\right) \exp \left(-\sum_{j=2}^{k-1} \frac{t r_{1}}{r_{j}}+\sum_{j=k+1}^{n-1} \frac{t r_{1}}{r_{j}}\right) d t
\end{aligned}
$$

Similarly,

$$
\left|\frac{f\left(z_{k}\right)}{z_{k}}\right| \leq \int_{0}^{1}(1-t) \prod_{\substack{j=1 \\ j \neq k}}^{n-1}\left|1-\frac{t z_{k}}{z_{j}}\right| d t
$$

Applying Lemma 4.2 with $r=r_{k}$ and $\rho=r_{j}$ when $1 \leq j \leq k-1$, and applying Lemma 4.3 with $r=r_{k}$ and $\rho=r_{j}$ when $k+1 \leq j \leq n-1$, we obtain 


$$
\begin{aligned}
\left|\frac{f\left(z_{k}\right)}{z_{k}}\right| & \leq \int_{0}^{1}(1-t) \prod_{j=1}^{k-1}\left(1+\frac{t r_{k}}{r_{j}}\right) \prod_{j=k+1}^{n-1}\left(1-\frac{t r_{k}}{r_{j}}\right) d t \\
& \leq \int_{0}^{1}(1-t)\left(1+\frac{t r_{k}}{r_{1}}\right) \exp \left(\sum_{j=2}^{k-1} \frac{t r_{k}}{r_{j}}-\sum_{j=k+1}^{n-1} \frac{t r_{k}}{r_{j}}\right) d t
\end{aligned}
$$

Now define $u=r_{k} / r_{1} \geq 1$ and

$$
A=\sum_{j=2}^{k-1} \frac{r_{k}}{r_{j}}-\sum_{j=k+1}^{n-1} \frac{r_{k}}{r_{j}}
$$

Any empty sum in the definition of $A$ is taken to be zero. In particular, $A=0$ if $n=3$. With $h_{1}(u, A)$ and $h_{2}(u, A)$ defined as in (4.1) and (4.2), we see that

$$
\left|\frac{f\left(z_{1}\right)}{z_{1}}\right| \leq h_{1}(u, A), \quad\left|\frac{f\left(z_{k}\right)}{z_{k}}\right| \leq h_{2}(u, A) .
$$

By Lemma 4.1, we obtain (4.3).

The above proof, together with the discussion of the cases of equality in Lemma 4.1, shows that we have strict inequality in (4.3) except possibly when $n=3$ and $r_{1}=r_{k}$. However, even then we have strict inequality in (4.4) and (4.5) when $0<t \leq 1$. We conclude that we have strict inequality in (4.3) in all cases.

This completes the proof of Theorem 1.3.

\section{References}

[1] A. F. Beardon, D. Minda and T. W. Ng, 'Smale's mean value conjecture and the hyperbolic metric', Math. Ann. 322 (2002), 623-632.

[2] A. Conte, E. Fujikawa and N. Lakic, 'Smale's mean value conjecture and the coefficients of univalent functions', Proc. Amer. Math. Soc. 135 (2007), 3295-3300.

[3] A. Córdova and S. Ruscheweyh, 'Subordination of polynomials', Rocky Mountain J. Math. 21 (1991), 159-170.

[4] E. Crane, 'Extremal polynomials in Smale's mean value conjecture', Comput. Methods Funct. Theory 6 (2006), 145-163.

[5] E. Crane, 'A bound for Smale's mean value conjecture for complex polynomials', Bull. Lond. Math. Soc. 39 (2007), 781-791.

[6] V. N. Dubinin, 'Inequalities for critical values of polynomials', Sb. Math. 197 (2006), 1167-1176.

[7] E. Fujikawa and T. Sugawa, 'Geometric function theory and Smale's mean value conjecture', Proc. Japan Acad. Ser. A Math. Sci. 82 (2006), 97-100.

[8] A. Hinkkanen and I. Kayumov, 'On critical values of polynomials with real critical points', Constr. Approx., to appear.

[9] P. Marinov and B. Sendov, 'Verification of the Smale's mean value conjecture for $n \leq 10$ ', C. $R$. Acad. Bulgare Sci. 60 (2007), 1151-1156.

[10] Q. I. Rahman and G. Schmeisser, Analytic Theory of Polynomials (Oxford University Press, Oxford, 2003).

[11] T. Sheil-Small, Complex Polynomials (Cambridge University Press, Cambridge, 2002).

[12] M. Shub and S. Smale, 'Computational complexity: on the geometry of polynomials and a theory of cost: II', SIAM J. Comput. 15 (1986), 145-161. 
[13] S. Smale, 'The fundamental theorem of algebra and complexity theory', Bull. Amer. Math. Soc. (N.S.) 4 (1981), 1-36.

[14] D. Tischler, 'Critical points and values of complex polynomials', J. Complexity 5 (1989), 438-456.

AIMO HINKKANEN, Department of Mathematics, University of Illinois at Urbana-Champaign, 1409 West Green Street, Urbana, IL 61801, USA

e-mail: aimo@illinois.edu

ILGIZ KAYUMOV, Institute of Mathematics and Mechanics, Kazan State University, Kremlevskaya 18, 420008 Kazan, Russia

e-mail: Ilgis.Kayumov@ksu.ru 